\title{
Avidity of anti-P aeruginosa antibodies during chronic infection in patients with cystic fibrosis
}

\author{
Oana Ciofu, Tine D Petersen, Per Jensen, Niels Høiby
}

\begin{abstract}
Background-In order to study the impact on the lung function of patients with cystic fibrosis of the avidity of antipseudomonal antibodies, the avidity of antibodies against the chromosomal $\beta$-lactamase of Pseudomonas aeruginosa (aßab) and against the 60-65 kDa heat shock protein of $P$ aeruginosa (antiGroEL) were measured in serum samples collected longitudinally during chronic infection with $P$ aeruginosa from a group of patients with poor and good lung function.
\end{abstract}

Methods-The thiocyanate elution method in which the molarity of potassium thiocyanate required to elute $50 \%$ bound antibody under conditions of antigen excess in ELISA was used to measure the relative avidity.

Results-All patients developed increasing levels of $\mathrm{a} \beta \mathrm{ab}$ and anti-GroEL antibodies during the follow up period but no maturation of the avidity of these antibodies was observed. In patients with good lung function the avidity of aßab was higher than in patients with poor lung function $(p=0.018)$. No significant difference in the avidity of the anti-GroEL antibodies was observed between the two groups of patients.

Conclusion-In patients with cystic fibrosis a high avidity of aßab could contribute to a more efficient inhibition of the $\beta$-lactamase by these antibodies, resulting in the better lung function seen in this group. The immunopathological implication of the failure in avidity maturation of antibodies in chronic infection is discussed.

(Thorax 1999;54:141-144)

Keywords: antibody avidity; Pseudomonas aeruginosa; cystic fibrosis

Department of Clinical Microbiology, Danish

Cystic Fibrosis Centre, Rigshospitalet, Copenhagen, Denmark N Høiby

Correspondence to: Dr O Ciofu, Panum Institute, IMMI 24.1, Blegdamsvej 3, 2200 Copenhagen N, Denmark.

Received 5 May 1998 Returned to author 30 June 1998

Revised manuscript received 12 August 1998 Accepted for publication

11 September 1998 aeruginosa antigens that are not able to clear the infection but instead correlate with a poor prognosis. ${ }^{34}$ The correlation between the antibody response and poor prognosis has been shown to be due to chronic inflammation in the lungs of patients with cystic fibrosis which is immune complex mediated. ${ }^{5}$ It has furthermore been found that the serum of patients with cystic fibrosis has a defective functional capacity, ${ }^{6-13}$ suggesting that the antipseudomonal antibodies in patients with cystic fibrosis might be of low avidity.

To address the question of whether the avidity of the antipseudomonal antibodies influences lung function we have measured the avidity of antibodies against the chromosomal $\beta$-lactamase of $P$ aeruginosa ( $\mathrm{a} \beta \mathrm{ab}$ ) and against the $60-65 \mathrm{kDa}$ heat shock protein of $P$ aeruginosa (anti-GroEL) in groups of patients with poor and good lung function.

\section{Methods}

PATIENTS

The diagnosis of cystic fibrosis was based on the accepted criteria including genotype, abnormal sweat electrolyte levels in repeated tests, and exocrine pancreatic insufficiency. After the diagnosis the patients were monitored during monthly visits to the Danish Cystic Fibrosis Centre at Rigshospitalet where clinical data and bacteriology of bronchopulmonary infections have been recorded prospectively since 1970.

Pulmonary function (forced vital capacity (FVC) and forced expiratory volume in one second $\left(\mathrm{FEV}_{1}\right)$ ) were determined at each monthly visit using an electronic spirometer (Spirotron, Dräger, Denmark). All values were expressed as percentage of the expected values according to height and sex. The individual values in this study were the mean of all results during one year of the observation period. For practical purposes the duration of the chronic infection was expressed in seven observation periods: $1-2,2-3,3-4,4-5,5-7,7-9,9-11$ years from the onset of the chronic infection. Patients with poor/good lung function were considered those with $\mathrm{FEV}_{1} \leqslant 70 \% / \mathrm{FEV}_{1}$ $>70 \%$ in at least six of the seven observation periods of the chronic infection. In the last observation period the mean (SD) body mass index was $20.8(2.4) \mathrm{kg} / \mathrm{m}^{2}$ in the group of patients with good lung function and 18.5 (2.7) $\mathrm{kg} / \mathrm{m}^{2}$ in those with poor lung function.

Multiple serum samples (eight/patient) from 11 patients with poor lung function and nine with good lung function were analysed for levels and avidity of $\mathrm{a} \beta \mathrm{ab}$ and anti-GroEL IgG antibodies. The patients were matched for age 

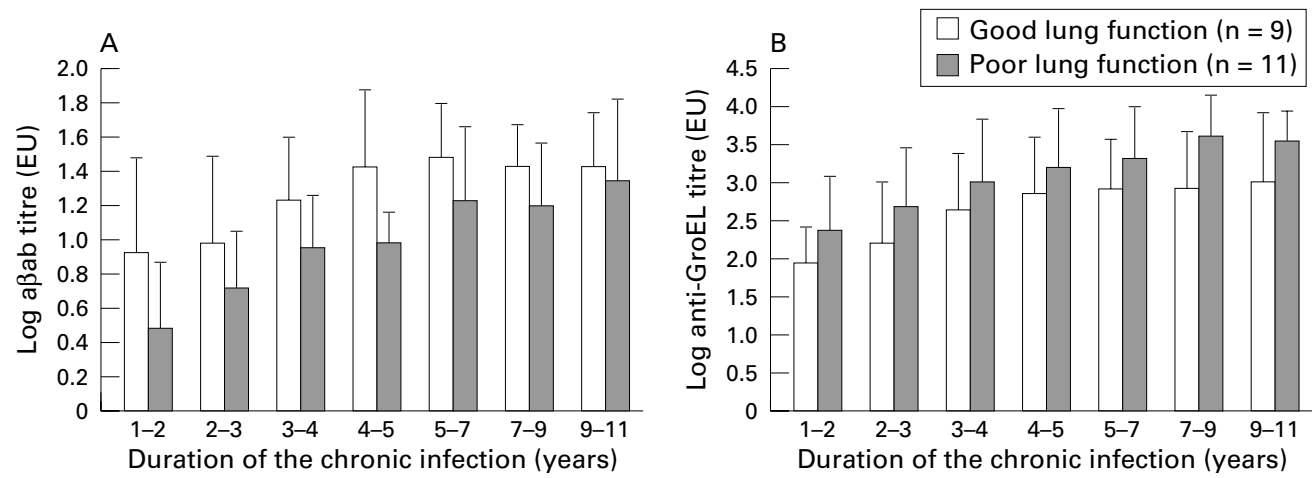

Figure 1 Development of serum $\operatorname{Ig} G(A)$ anti- $\beta$-lactamase antibodies and $(B)$ anti-GroEL antibodies during 11 years of chronic infection with $P$ aeruginosa in nine patients with good lung function and 11 patients with poor lung function. The data were log transformed and are presented as means with $95 \%$ confidence intervals.

at the onset of the chronic infection (mean 10 years (range 0.8-29)) and for treatment with $\beta$-lactam antibiotics.

Since 1976 all the chronically infected cystic fibrosis patients have been admitted every third month at the Danish Cystic Fibrosis Centre for a two week intravenous course of antipseudomonal antibiotics. The treatment consisted of a combination of tobramycin and a $\beta$-lactam antibiotic. ${ }^{14}$ All 20 patients had $P$ aeruginosa strains expressing $\beta$-lactamase mediated resistance to $\beta$-lactam antibiotics, as previously shown. ${ }^{15-17}$

AVIDITY OF SERUM IgG A $\beta$ AB AND ANTI-GroEL ANTIBODIES

The avidity of IgG a $\beta a b$ and anti-GroEL antibodies was estimated by a thiocyanate elution enzyme immunoassay as previously described ${ }^{18}$ in which the molarity of the thiocyanate required to elute $50 \%$ bound antibody under conditions of antigen excess was used as a measure of relative avidity. Preliminary experiments showed that there was no significant elution of solid phase bound $\beta$-lactamase or GroEL at any of the molarities of thiocyanate used in the subsequent assays.

Microtitre plates (Microwell, Nunc, Tåstrup, Denmark) and reagent volumes of $100 \mu \mathrm{l}$ were used. Plates were coated with $2 \mu \mathrm{g} /$ $\mathrm{ml}$ purified $\beta$-lactamase or recombinant GroEL in $0.1 \mathrm{M}$ carbonate buffer, $\mathrm{pH}$ 9.8, overnight (at least 15 hours) at $4^{\circ} \mathrm{C}$ on a shaking table as previously reported. ${ }^{17}{ }^{19}$ Each serum sample was assayed in 19 replicate wells. Sixteen wells (eight duplicates) were used for the affinity elution procedure with eight different molarities of the KSCN and three wells (one triplicate) for the control without KSCN. After the affinity elution procedure (see next section), horseradish peroxidase-labelled rabbit anti-human IgG, P214, (Dako, Denmark) was added to each well. The optical density (OD) at $492 \mathrm{~nm}$ was read using an automatic plate reader. Two-fold dilutions of a pool of 10 cystic fibrosis serum samples selected from the late phases of infection with high antibody titres were used to construct standard curves for $\mathrm{a} \beta \mathrm{ab}$ and anti-GroEL antibodies. The $\mathrm{a} \beta \mathrm{ab}$ and anti-GroEL antibody titres of the control samples without KSCN were tested on each microtitre plate. For the $a \beta a b$ ELISA an arbitrary value of 100 ELISA units (EU) was given to the 1:1000 dilution of the pool, and for the antiGroEL antibody ELISA an arbitrary value of 20000 ELISA units was given to the 1:20 000 dilution of the pool. Multiple regression was used to predict from the standard curve the antibody concentration (in EU) corresponding to the OD values of the control tested samples (mean OD of triplicate determinations in the absence of KSCN).

\section{AFFINITY ELUTION PROCEDURE}

Potassium thiocyanate (KSCN) was added to the duplicated test samples $(100 \mu l$ per well $)$ at concentrations of $0.5,0.75,1,1.5,2,3,4,5 \mathrm{M}$ in the dilution buffer. This range of KSCN concentrations was chosen after pilot experiments using concentrations from 0.1 to $8 \mathrm{M}$. In two serum samples with high avidity, KSCN molarities of $6 \mathrm{M}$ and $8 \mathrm{M}$ were used. The plates were incubated for 30 minutes prior to washing and addition of the conjugate for ELISA.

\section{DATA ANALYSIS}

The absorbance readings in the absence of thiocyanate were assumed to represent effective total binding of specific antibody, and subsequent absorbance readings in the presence of increasing concentrations of thiocyanate were converted to the appropriate percentage of the total bound antibody. ${ }^{18}$ The data were fitted to a graph of $\log _{10}$ (percentage binding) versus molar concentration of KSCN by linear regression analysis, and the affinity index, representing the molar concentration of thiocyanate required to reduce the initial optical density by $50 \%$, was estimated. Data were rejected as unreliable if the initial absorbance was below 0.2 or if the correlation coefficient for the linear fitting was below 0.80 .

REPRODUCIBILITY OF ASSAYS

Ten serum samples from cystic fibrosis patients with chronic $P$ aeruginosa infection were tested on two different plates on two different days. The intra-plate, plate to plate, and day to day variations of the avidity index were determined using the formula $\mathrm{SD}=\sum \mathrm{d}^{2} / 2 n$ where SD is the standard deviation, $\sum \mathrm{d}^{2}$ is the sum squared differences of double determinations of the same sample, and $n$ is the number of observations. 

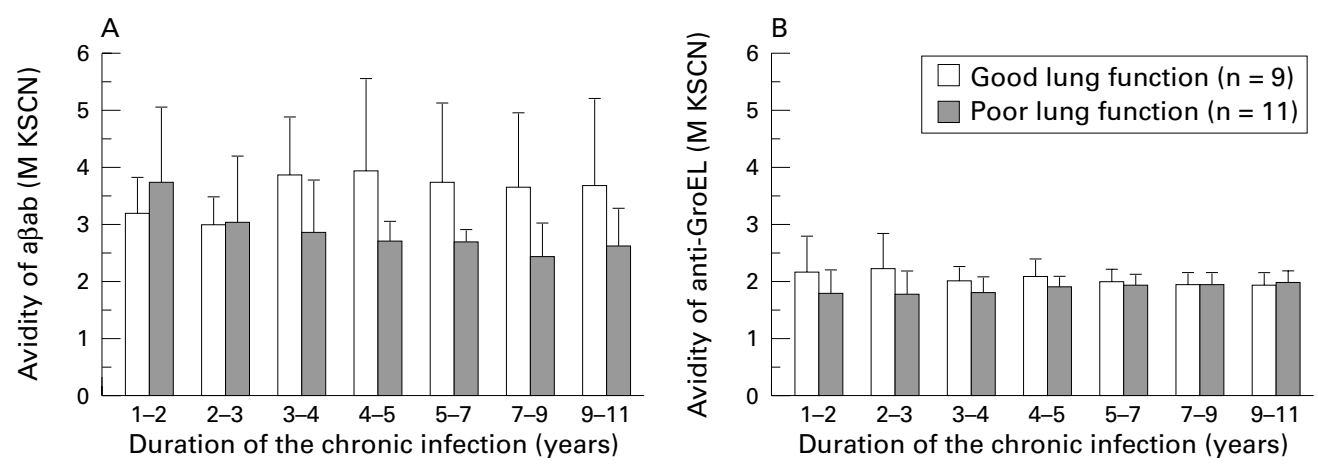

Figure 2 Avidity of serum $\operatorname{IgG}(A)$ anti-B-lactamase antibodies and (B) anti-GroEL antibodies during 11 years of chronic infection with $P$ aeruginosa in nine patients with good lung function and 11 patients with poor lung function. The data are presented as means with $95 \%$ confidence intervals.

For the intraplate variations four different KSCN molarities were used on each plate. For the a $\beta \mathrm{ab}$ ELISA the intra-plate, plate to plate, and day to day variations of the avidity index were $1.1 \%, 2.7 \%$, and $2.8 \%$, respectively, and for the GroEL ELISA they were $1.2 \%, 2.95 \%$, and $3.4 \%$, respectively.

To test the validity of our assay we measured the avidity of anti-GroEL antibodies in three patients without cystic fibrosis but with $P$ aeruginosa infection and we found, as expected, that the avidity index increased in consecutive serum samples (results not shown).

STATISTICAL ANALYSIS

The analysis of variance (ANOVA) test for repeated measures was used to analyse the differences that occurred in time between the two groups of patients. Logarithmic transformation of the $a \beta a b$ and anti-GroEL titres was performed to assure a Normal distribution of the data. The StatView 5.1 software was used for statistical description of the results. The level of significance of the test was $5 \%$.

\section{Results}

The development of IgG a $\beta a b$ and anti-GroEL antibody titres during the first 11 years of the chronic infection in 11 cystic fibrosis patients with poor lung function and nine with good lung function is shown in fig 1 . In both groups of patients there was a significant increase with time in the serum IgG antibody titres against $\beta$-lactamase and GroEL ( $p<0.0001)$. Patients with poor lung function had higher titres of anti-GroEL antibodies and lower titres of $a \beta a b$ than those with good lung function, although the difference between the groups did not reach statistical significance.

The avidity of serum IgG $\mathrm{a} \beta \mathrm{ab}$ and antiGroEL antibodies in the two groups of patients is shown in fig 2 .

No maturation in the avidity of $a \beta a b$ and anti-GroEL antibodies was observed during chronic infection with $P$ aeruginosa.

Patients with good lung function had $a \beta a b$ antibodies of significantly higher avidity than those with poor lung function $(p=0.018)$. There was no significant difference in the avidity of anti-GroEL antibodies between the two groups of patients. The relative avidity of the anti-GroEL antibodies was lower than the avidity of the $\mathrm{a} \beta \mathrm{ab}$ at all points in time.

\section{Discussion}

After immunisation, B cells producing high affinity antibodies are selected in the germinal centres of the lymph nodes and this leads to a maturation in time of the avidity of the antibodies. ${ }^{20} \mathrm{~A}$ chronic course of infection represents a different situation as large amounts of antigen are permanently available. A reduction in the affinity of $\operatorname{IgG}_{1}$ antibodies after a long exposure to an antigen via the gut has been observed previously. ${ }^{21}$ The failure in avidity maturation of antibodies in chronic infection might have immunopathological implications as antigen-antibody complexes containing low avidity antibodies are not readily cleared by the immune system. ${ }^{2}$

In the present study we addressed the question of whether the lung function of patients with cystic fibrosis may be influenced by the avidity of the antipseudomonal antibodies as it is known that chronic inflammation in the lungs of patients with cystic fibrosis is immune complex mediated. ${ }^{5}$ The levels and avidity of the IgG a $\beta a b$ and anti-GroEL antibodies were measured in 11 cystic fibrosis patients with poor lung function and nine with good lung function. Although the levels of a $\beta a b$ and antiGroEL antibodies increased in both groups of patients during the 11 year follow up period, no avidity maturation of these antibodies was observed. This is in agreement with studies by Schaad, ${ }^{22}$ Bruderer, ${ }^{12}$ Lang, ${ }^{13}$ and Pier ${ }^{10}$ who found that the anti- $P$ aeruginosa antibodies induced by chronic infection in patients with cystic fibrosis are of low avidity and low opsonophagocytic capacity compared with vaccination induced antibodies.

The avidity pattern was different for the two different antigens in the two cohorts of patients. While patients with good lung function had a $\beta \mathrm{ab}$ antibodies of significantly higher avidity than patients with poor lung function, no significant difference in the avidity of the anti-GroEL antibodies was observed between the two groups of patients. The different pattern in the avidity maturation of $a \beta a b$ and anti-GroEL antibodies might be due to differences in the IgG subclass response against the two antigens as it has been shown that the different human IgG subclasses express different avidities. ${ }^{212324}$

In conclusion, the avidity of antipseudomonal antibodies does not seem to mature during 
the course of chronic infection with $P$ aeruginosa in patients with cystic fibrosis, which could be responsible in part for the progressive destruction of the lungs mediated by immune complexes. However, patients with cystic fibrosis with good lung function have higher avidity $\mathrm{a} \beta \mathrm{ab}$ than those with poor lung function, which may contribute to a more efficient inhibition of the $\beta$-lactamase by these antibodies and subsequently result in a better effect of the $\beta$-lactam antibiotics.

The authors wish to thank Tina Wasserman for excellent technical assistance. This study was partially supported by the Danish Medical Research Council.

1 Steward MW. The biological significance of antibody affinity. Immunol Today 1981;July:134-40.

2 Devey ME, Bleadsdale K, Stanley C, et al. Failure of affinity maturation leads to increased suceptibility to immune complex glomerulonephritis. Immunology 1984;52:377-83.

3 Høiby N. Pseudomonas aeruginosa infection in cystic fibrosis Diagnostic and prognostic significance of Pseudomonas aeruginosa precipitins determined by means of crossedimmunoelectrophoresis. A survey. APMIS, Section C 1977; 262 (Suppl):3-96

4 Pedersen SS. Lung infection with alginate-producing, mucoid Pseudomonas aeruginosa in cystic fibrosis patients. APMIS 1992;Suppl 28 .

5 Høiby N, Döring G, Schiøtz PO. The role of immune complexes in the pathogenesis of bacterial infection. Annu Rev Microbiol 1986;40:29-53.

6 Moss RB, Lewiston NJ. Immune complexes and humoral response to Pseudomonas aeruginosa in cystic fibrosis. Am response to Pseudomonas aeru.

7 Eichler I, Joris L, Hsu Y-P, et al. Nonopsonic antibodies in cystic fibrosis. Pseudomonas aeruginosa lipopolysaccharidespecific immunoglobulin $G$ antibodies from infected patient sera inhibit neutrophil oxidative responses. F Clin Invest 1989;84:1794-804.

8 Fick RB, Naegel GP, Matthay RA, et al. Cystic fibrosis pseudomonas opsonins. Inhibitory nature in an vitro phagocytic assay. $\mathcal{F}$ Clin Invest 1981;68:899-914

9 Thomassen MJ, Demko CA, Wood RE, et al. Ultrastructure and function of alveolar macrophages from cystic fibrosis patients. Pediatr Res 1980;14:715-21.

10 Pier G, Grout M, Desjardins D. Complement deposition by antibodies to Pseudomonas aeruginosa mucoid polysaccharide (MEP) and non-MEP specific opsonins. F Immunol 1991;147:1869-76.

11 Pressler T, Jensen ET, Esperesen F, et al. Correlation between the specific IgG subclass antibodies to Pseu- domonas aeruginosa and opsonic activity in serum from patients with cystic fibrosis. Pediatr Pulmonol 1994;17:3140

12 Bruderer U, Cryz SJ, Schaad UB, et al. Affinity constants of naturally acquired and vaccine-induced anti-Pseudomonas aeruginos a antibodies in healthy adults and cystic fibrosis patients. F Infect Dis 1992;166:344-9.

13 Lang A, Schaad U, Rüdeberg A, et al. Effect of high-affinity anti-Pseudomonas aeruginosa lipopolysaccharide antibodies induced by immunization on the rate of Pseudomonas aeruginosa infection in patients with cystic fibrosis. $\mathcal{F}$ Pediatr 1995;127:711-7.

14 Jensen T, Koch C, Høiby N. Use of antibiotics in cystic fibrosis. Antibiot Chemother 1989;42:237-46.

15 Giwercman B, Lambert P, Rosdahl VT, et al. Rapid emergence of resistance in Pseudomonas aeruginosa cystic fibrosis patients due to in vivo selection of stable partially derepressed $\beta$-lactamase producing strains. I Antimicrob Chemother 1990;26:247-59.

16 Petersen TD, Ciofu O, Pressler T, et al. Quantitative analysis of the IgG and IgG subclass immune responses to chromosomal Pseudomonas aeruginosa $\beta$-lactamase in sera from cystic fibrosis patients by means of western-blotting and laser scanning densitometry. Thorax 1996;51:733-8.

17 Ciofu O, Giwercman B, Rassmusen JW, et al. Antibodies against chromosomal Pseudomonas aeruginosa $\beta$-lactamase are markers of the development of resistance of $P$ aeruginosa in patients with cystic fibrosis. F Antimicrob Chemother 1995;35:295-304.

18 Pullen GR, Fitzgerald MG, Hosking CS. Antibody avidity determination by ELISA using thiocyanate elution. 7 Immunol Methods 1986;86:83-7.

19 Jensen P, Høiby N, Lanng S, et al. Increased level of anti- $P$ aeruginosa GroEL antibodies prior to onset of diabetes mellitus in patients with cystic fibrosis. APMIS (In press).

20 Gearhart P. Somatic mutation and affinity maturation. In: William EP, ed. Fundamental immunology. New York: Raven Press, 1993: 865-85.

21 Devey ME, Beckman S, Kemeny DM. The functional affinities of antibodies of different IgG subclasses to dietary antigens in mothers and their babies. Clin Exp Immunol 1993;94:117-21.

22 Schaad UB, Lang AB, Wedgwood J, et al. Serotype-specific serum IgG antibodies to lipopolysaccharides of Pseudomonas aeruginosa in cystic fibrosis: correlation to disease, subclass distribution, and experimental protective capacity. Pediatr Res 1990;27:508-13.

23 Devey ME, Bleasdale-Barr KM, Bird P, et al. Antibodies of different human IgG subclasses show distinct patterns of affinity maturation after immunization with keyhole limpet haemocyanin. Immunology 1990;70:168-74.

24 McCloskey N, Turner MW, Steffner P, et al. Human constant regions influence the antibody binding characteristics of mouse-human chimeric IgG subclasses. Immunology 1996;88:169-73. 